\title{
Avaliação de cesáreas na Maternidade Escola Assis Chateaubriand utilizando o sistema de classificação de Robson em dez grupos
}

\section{Evaluation of cesarean births in Maternidade Escola Assis Chateaubriand using the Robson ten group classification system}

\begin{abstract}
Victor de Alencar Mouraํ. Francisco Edson de Lucena Feitosa².
1 Médico, ginecologista e obstetra, aluno do Mestrado Profissional em Saúde da Mulher e da Criança, da Faculdade de Medicina da Universidade Federal do Ceará (UFC), Fortaleza, Ceará, Brasil. 2 Médico, ginecologista e obstetra, Doutorado em Tocoginecologia pela Universidade Estadual de Campinas, professor do Departamento de Saúde Materno-Infantil, Maternidade Escola Assis Chateaubriand (MEAC), Universidade Federal do Ceará (UFC), Fortaleza, Ceará, Brasil.
\end{abstract}

\section{RESUMO}

Introdução: o parto cesárea é aconselhado quando não é provável que se realize o parto vaginal com segurança. Observa-se que no Brasil a taxa de cesarianas na rede pública é de aproximadamente 52\%. Objetivo: esta pesquisa teve como objetivo a coleta de informações referentes a partos cesárea realizados na Maternidade Escola Assis Chateaubriand no período de março a julho de 2015, considerando a importância de uma base para a indicação de cesáreas e o propósito de reduzir o número deste procedimento. Métodos: foram considerados 100 partos de cada mês, escolhidos aleatoriamente, contabilizando 500 partos cesárea de um total de 2230 partos realizados no período. Os dados coletados foram avaliados considerando o Sistema de Classificação de Robson em Dez Grupos (SCRDG). Este sistema de classificação baseia-se em características obstétricas das pacientes, considerando as semelhanças. Resultados: observou-se que no período avaliado a taxa de cesáreas foi de $53,7 \%$. O Grupo 5 foi o que mais contribuiu para a taxa de cesáreas $(25,2 \%)$, seguido pelo Grupo 2 (18,6\%). Conclusão: este resultado deve ser considerado no planejamento da unidade hospitalar bem como com relação às políticas públicas, fornecendo subsídios para que se possa sugerir medidas para reduzir a frequência de cesáreas no referido hospital.

Palavras-chave: Cesárea. Saúde materna. Atenção terciária à saúde.

\section{ABSTRACT}

Introduction: the cesarean birth is advised when it is not possible to do the vaginal birth with assurance. In Brazil, the rate of cesarean sections in the public hospital network it is around $52 \%$. Objective: the objective of this research was to collect data about cesarean births made in Maternidade Escola Assis Chateaubriand during March to July 2015, considering the importance of a study to give support for the indication of cesarean section and the purpose to reduce the number of this procedure. Methodology: a total of 500 cesarean sections were evaluated, randomly selected, considering that in this period 2,230 childbirths were made. The collected data were evaluated considering the Robson Ten Group Classification System (RTGCS). This classification system is based on obstetric characteristics of patients, taking into account the similarities between them. Results: the results showed that the cesarean rate was $53.7 \%$ in the studied interval. Group 5 was the one with the greater contribution to cesarean deliveries (25.2\%), followed by Group $2(18.6 \%)$. Conclusion: this result should be considered in the hospital planning as well in relation to public policies, providing subsidies to suggest actions to reduce the cesarean rate in this hospital.

Keywords: Cesarean section. Maternal health. Tertiary healthcare.

Autor correspondente: Victor de Alencar Moura, Rua Professor Costa Mendes, 1608, $5^{\circ}$ andar, Rodolfo Teófilo, Fortaleza, Ceará. CEP: 60416-200. Telefone: +55 84 98865-2206. E-mail: victordealencar@hotmail.com

Conflito de interesses: Não há qualquer conflito de interesses por parte de qualquer um dos autores.

Recebido em: 05 Mai 2016; Revisado em: 29 Mai 2016; Aceito em: 07 Jun 2016. 


\section{INTRODUÇÃO}

O parto cesárea, ou cesariana, consiste na extração do feto através de uma incisão na parede abdominal (laparotomia) e na parede uterina (histerotomia). Durante sua evolução, a cesariana teve diversos significados, considerando a época e a civilização. Inicialmente, ela tinha como objetivo a retirada da criança da mãe morta ou em estado terminal, visando salvar a criança ou para sepultá-la separadamente da mãe, como exigiam algumas religiões. ${ }^{1}$

Josipović, Stojkanović e Brković ${ }^{2}$ relatam que as indicações obstétricas mais comuns para cesariana em meados do século 20 eram: hemorragia devido ao descolamento da placenta, assinclitismo e desproporção céfalo-pélvica. Atualmente, as indicações mais comuns são: uma cesariana anterior, distócia, apresentação pélvica e sofrimento fetal. Estes autores relatam que o parto vaginal está se tornando menos desejado pelas parturientes, o que faz com que a demanda por cesarianas aumente.

De acordo com a OMS, ${ }^{3}$ a comunidade internacional de saúde tem considerado como ideal uma taxa de cesáreas entre $10 \%$ e $15 \%$ de todos os partos. No Brasil, baseado no estudo realizado pela Fiocruz, estima-se em $52 \%$ o total de cesáreas e, considerando apenas a rede privada, este número chega a $88 \% .{ }^{4}$ De acordo com esta pesquisa, cerca de um milhão de mulheres são submetidas anualmente a cesarianas sem indicação obstétrica.

Considerando este aumento dos partos abdominais, Patah e Malik $^{5}$ realizaram uma revisão da literatura e verificaram que as principais justificativas para este procedimento são: fatores sociais, demográficos, culturais e econômicos das gestantes; solicitação materna pelo tipo de parto; fatores relacionados ao modelo assistencial desenvolvido no país, que envolvem aspectos do trabalho médico e de outros profissionais; e preferências médicas.

O profissional de saúde e suas orientações são de grande importância para a escolha da via de parto pela gestante, principalmente as primigestas, o que é evidenciado pela diferença na quantidade de partos por via cesárea entre os setores público e privado. ${ }^{6} \mathrm{O}$ médico e o sistema de saúde devem, em conjunto, buscar identificar quais as razões que levam as mães a solicitar o parto por via cesárea. Uma vez que estes motivos forem identificados, deve-se verificar se há realmente indicativos que justifiquem essa via de parto, além de justificar sua necessidade ou não. É importante considerar que, para fazer uma correta avaliação da necessidade de indicação de cesáreas, deve-se buscar um aumento dos melhores resultados para a mãe e para o bebê, fazendo considerações sobre os recursos de saúde disponíveis e a preferência da gestante. ${ }^{7}$

De acordo com Dias et al., ${ }^{8}$ as taxas de cesarianas não devem ser classificadas como altas ou baixas, mas como necessárias e desnecessárias. Visando, portanto, à redução do número de cesarianas desnecessárias, ressalta-se a importância de se melhorar a qualidade da coleta de informações de rotina em todos os aspectos do parto. Concomitantemente, percebe-se a relevância de se adotar sistemas de classificação padrão para que possam ser feitas comparações e, a partir de então, sejam estabelecidas medidas para melhorar o atendimento., ${ }^{9,10}$ Estes sistemas de classificação são aplicados na área médica com o intuito de transformar dados brutos em informações úteis para melhorar o atendimento clínico.

Abden-Aleen et al. ${ }^{11}$ ressaltam que é crucial que se tenha um sistema padrão para classificação de indicações de cesáreas. Este sistema deve ter sido aplicado por longo tempo e deve ser aceito em níveis nacionais e internacionais. De acordo com estes autores, o sistema de classificação ideal deve ser simples de aplicar e fácil de compreender, e suas categorias devem ser clinicamente relevantes. Este sistema deve possibilitar comparações entre centros, nações e políticas diferentes de gestão obstétrica, fornecendo subsídios para a análise precisa das implicações da cesariana, tanto em curto como em longo prazos.

Robson, em 2001, apresentou um novo sistema de classificação, o Sistema de Classificação de Robson em Dez Grupos (SCRDG). Os agrupamentos são definidos de tal forma que eles são mutuamente exclusivos e totalmente inclusivos, em que todos os dados podem ser incluídos nos grupos. ${ }^{9}$ Em 2015, a Organização Mundial de Saúde lançou o documento intitulado "Declaração da OMS Sobre Taxas de Cesáreas". Neste documento eles propõem a utilização mundial da Classificação de Robson como instrumento padrão de avaliação, comparação e monitoramento das taxas de cesáreas. ${ }^{3}$

Betrán et al. $^{12}$ fizeram uma revisão sistemática sobre a Classificação de Robson, considerando os atores envolvidos no processo e de que forma ela pode ser aprimorada. Nesta revisão eles identificaram 73 artigos científicos que descreviam as experiências dos usuários sobre os prós e contras da sua adoção, implementação e interpretação dos resultados. De acordo com os usuários, os pontos fortes da classificação de Robson são a simplicidade do seu arcabouço, a validade do seu propósito, sua facilidade de implementação e a forma direta da interpretação inicial. A flexibilidade da classificação permite a criação de subdivisões em cada grupo, que podem melhorar as análises das práticas clínicas locais.

$\mathrm{Na}$ Maternidade Escola Assis Chateaubriand (MEAC), levando em conta os dados disponíveis em seus relatórios de gestão, constata-se que de 2011 a 2015 a média de partos cesárea foi de $49,36 \%$. Considerando o exposto, bem como o crescente número de indicações de cesáreas e a inexistência de estudo semelhante na referida maternidade, o objetivo desta pesquisa foi analisar parte da população obstétrica que passou por cesariana no período de março a julho de 2015. Utilizou-se o Sistema de Classificação de Robson em Dez Grupos (SCRDG) para identificar os grupos específicos de mulheres que mais contribuíram para o aumento da taxa de partos cesárea. A utilização do SCRDG irá ajudar na decisão da via de parto quando houver dúvidas e possibilitará a 
comparação entre centros distintos, fornecendo subsídios para a análise precisa das implicações da cesariana, tanto em curto como em longo prazo.

\section{METODOLOGIA}

\section{Local do estudo}

O estudo foi realizado no Alojamento Conjunto da MEAC, da Universidade Federal do Ceará. Este é um hospital terciário com cerca de 4000 partos por ano, sendo que deste total aproximadamente $49,3 \%$ são cesáreas.

\section{Definição da amostra}

Foram utilizados dados de prontuários referentes às internações para realização de parto cesárea durante o período de março a julho de 2015. No período foram realizadas 1197 cesáreas. Para este estudo selecionou-se, aleatoriamente, 100 prontuários por mês, totalizando 500 prontuários.

\section{Sistema de Classificação de Robson em Dez Grupos (SCRDG)}

Sistema apresentado por Robson em 2001 que tem como base cinco características distintas e incontestáveis de cada gravidez, nomeadamente: gravidez única ou múltipla; história obstétrica anterior (nulíparas e multíparas, com ou sem cesárea anterior); apresentação cefálica, pélvica ou córmica; início de trabalho de parto espontâneo ou induzido; e idade gestacional (prematuro ou a termo).Os agrupamentos são definidos de tal forma que eles são mutuamente exclusivos e totalmente inclusivos, em que todos os dados podem ser incluídos nos grupos., ${ }^{9} 13-18$

Para a captação dos dados e enquadramento da cesárea de acordo com o SCRDG, foi utilizada a ficha de coleta de dados mostrada na Figura 1. As pacientes foram identificadas somente por seu número de prontuário.

Figura 1. Ficha para classificar o parto de acordo com os grupos de Robson.

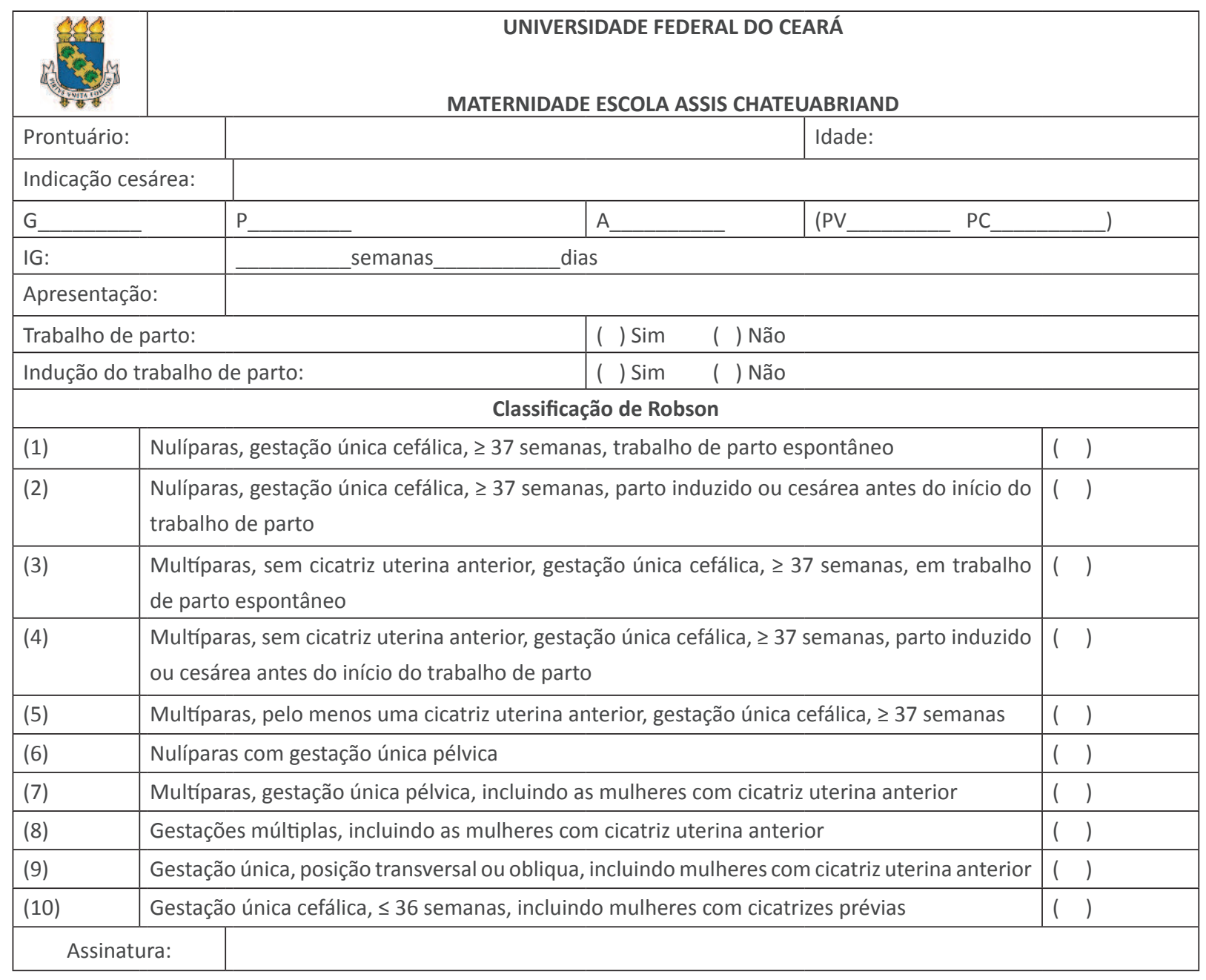

\section{Análise estatística}

Os dados foram consolidados utilizando o programa Excel (Microsoft $^{\circledR}$ 2010). As taxas de cesariana foram calculadas considerando os nascimentos em cada um dos 10 grupos do
SCRDG, durante o período estimado nesta pesquisa. Esta determinação foi feita dividindo o número de cesáreas no grupo pelo número total de cesáreas na população estudada. A contribuição de cada grupo de Robson foi avaliada para propor mudanças na taxa total de cesáreas. 


\section{Aspectos éticos}

O projeto de pesquisa seguiu todos os preceitos éticos que regem as pesquisas em seres humanos, respeitando a Portaria 466/12 do Conselho Nacional de Saúde ${ }^{19}$ e só teve início após aprovação pelo Comitê de Ética em Pesquisa envolvendo seres humanos da Maternidade Escola Assis Chateaubriand, da Universidade Federal do Ceará.

\section{RESULTADOS E DISCUSSÃO}

\section{Taxa de cesáreas}

De acordo com a Organização Mundial de Saúde ${ }^{20}$ não há justificativas plausíveis para que uma região apresente taxas de cesárea superiores a 10-15\%. Observou-se que no período avaliado as taxas de cesáreas foram superiores a $50 \%$, Gráfico 1 , o que está bem acima do valor sugerido pela OMS e de acordo com a estimativa da Fiocruz ${ }^{4}$ para partos cesárea no Brasil, que é de 52\%. Quando se avalia os dados considerando as médias do período para partos cesárea $(53,6 \%)$ e vaginal $(46,4 \%)$, percebe-se que o percentual de cesárea foi um pouco superior ao de parto vaginal, indicando a necessidade de uma análise detalhada das taxas de cesárea visando identificar os fatores que contribuíram para estes números.

Gráfico 1. Taxas de cesáreas para os meses avaliados.

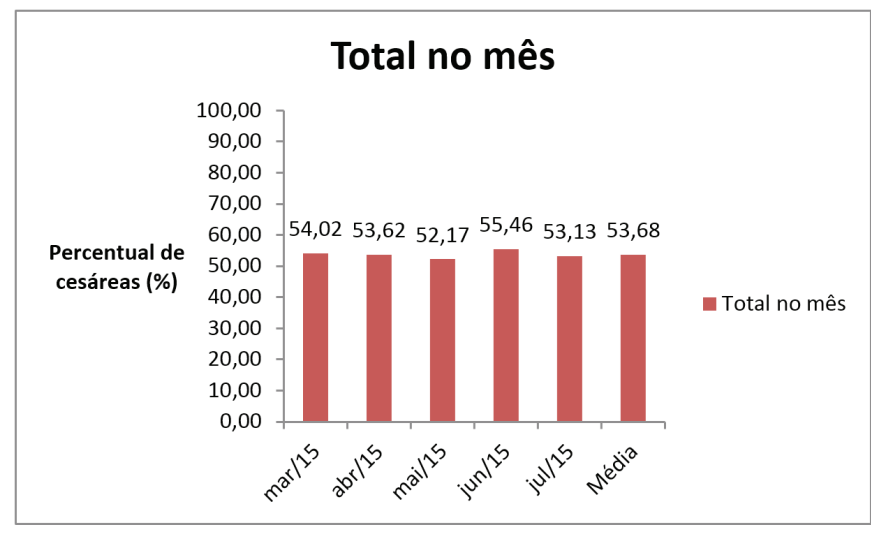

Ye et al. ${ }^{21}$ compilaram os dados referentes às últimas três décadas de 19 países, considerando: as taxas de cesáreas, os indicadores socioeconômicos e as taxas de mortalidade neonatal, infantil e materna. O objetivo da pesquisa foi identificar qual seria a taxa de cesárea ótima por recomendação médica. Eles observaram que quando as taxas de cesárea atingiam $10-15 \%$ as curvas de mortalidade tornavam-se quase horizontais, indicando que o aumento da taxa de cesáreas não tem impacto significativo sobre as taxas de mortalidade materna e infantil.

Conforme o exposto, verifica-se que há a necessidade de reduzir a taxa de cesáreas na MEAC. $\mathrm{O}$ estudo utilizando o SCRDG irá fornecer as informações necessárias para que, conhecendo os grupos que mais contribuíram para este valor, o corpo gestor possa tomar as medidas necessárias.

\section{Classificação de Robson}

As contribuições de cada grupo do SCRDG na taxa de cesáreas no período avaliado são apresentadas no Gráfico 2, que mostra as quantidades e os percentuais de cesáreas por grupo.

Gráfico 2. Distribuição das cesáreas no período de março a julho de 2015.

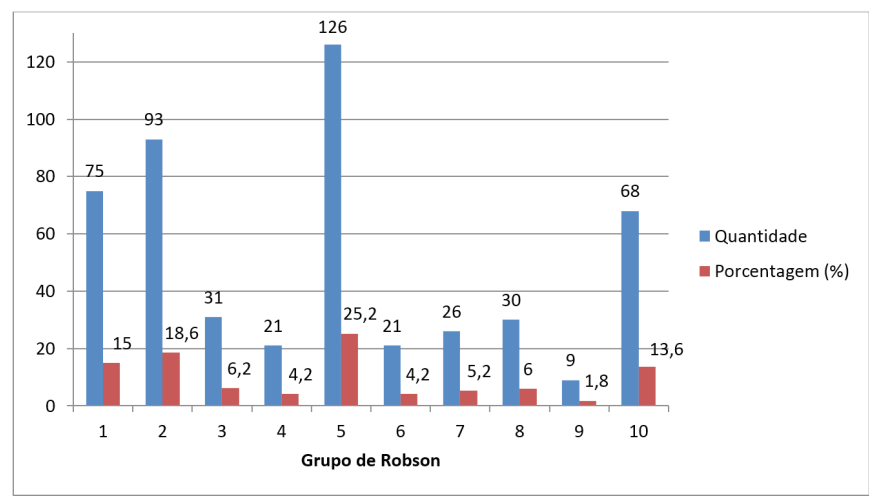

Conforme pode ser observado, as maiores contribuições foram dos grupos 5, 2, 1 e 10, respectivamente. O grupo 5 , correspondente ao grupo de parturientes a termo, fetos cefálicos, submetidas a cesariana anterior, apresenta o maior número de pacientes, o que está de acordo com a literatura pesquisada. ${ }^{11,12,16,17,21,22}$ Este dado é importante para que sejam tomadas medidas que reduzam a quantidade de cesarianas desnecessárias, com foco principalmente nas nulíparas a termo submetidas a cesarianas, com fetos em apresentação cefálica, após início espontâneo do trabalho de parto (grupo 1) ou após indução ou sem trabalho de parto (grupo 2). Permite, além disso, e elaboração de protocolos clínicos para que seja encorajada a realização de parto vaginal após cesariana, tendo em vista que as grandes taxas de cesárea nos grupos 1 e 2 afetam, no futuro, o grupo 5.

Com relação ao grupo 10 , que é o grupo de pacientes com 36 semanas ou menos, observa-se uma alta taxa de partos prematuros realizados no serviço, possivelmente por ser a MEAC a maternidade terciária de referência do estado do Ceará, recebendo as pacientes mais graves. Destaca-se a qualidade dos serviços de UTI materna e neonatal da referida maternidade, que, apesar das altas taxas de prematuridade e de gestantes de risco elevado, conseguem manter níveis baixos de mortalidade materna e neonatal, conforme a taxa de mortalidade hospitalar geral apresentada no relatório de gestão de $2015 .^{23}$

\section{CONCLUSÕES}

O desenvolvimento do estudo permitiu concluir que é importante implementar o SCRDG na MEAC, a fim de identificar e procurar soluções para obter taxas de cesáreas coerentes com o padrão da OMS. Verificou-se, ainda, que:

- O SCRDG é um instrumento simples, que pode ser facilmente utilizado para avaliar e propor medidas de controle da taxa de cesáreas; 
- Sua utilização permite a análise temporal dos dados, a identificação dos grupos de pacientes que mais contribuem para a taxa de cesáreas, e a comparação entre diferentes hospitais;

- O grupo 5 foi o que mais contribuiu para a taxa de

\section{REFERÊNCIAS}

1. Sewell JE. Cesarean section - a brief history [Internet]. Washington: American College of Obstetricians and Gynecologists; 1993 [acesso em: 03 Fev 2016]. Disponível em: https://www.nlm.nih.gov/ exhibition/cesarean/index.html

2. Josipović LB, Stojkanović JD, Brković I. Analysis of cesarean section delivery at Nova Bila Hospital according to the Robson Classification. Coll Antropol. 2015; 39(1):145-50.

3. Organização Mundial de Saúde. Declaração da OMS sobre taxas de cesáreas [Internet]. Genebra: OMS; 2015 [acesso em: 20 Jan 2016]. Disponível em: http://apps.who.int/iris/bitstream/10665/161442/3/ WHO_RHR_15.02_por.pdf

4. Fundação Osvaldo Cruz. Nascer no Brasil: inquérito nacional sobre parto e nascimento [Internet]. Rio de Janeiro: FIOCRUZ; Escola Nacional de Saúde Pública; 2015 [acesso em: 20 Jan 2016]. Disponível em: http://www.ensp.fiocruz.br/portal-ensp/informe/site/ arquivos/anexos/nascerweb.pdf

5. Patah LE, Malik AM. Modelos de assistência ao parto e taxa de cesárea em diferentes países. Rev Saude Publica. 2011;45(1):185-94.

6. Haddad SE, Cecatti JG. Estratégias dirigidas aos profissionais para a redução das cesáreas desnecessárias no Brasil. Rev Bras Ginecol Obstet. 2011;33(5):252-62.

7. NIH State-of-the-Science Conference Statement on cesarean delivery on maternal request. NIH Consensus and State-of-the-Science Statements. National Institutes of Health [Internet]. 2006 [acesso em: 20 Jan 2016];23(1):1-29. Disponível em: https://consensus.nih. gov/2006/cesareanstatement.pdf

8. Dias MA, Domingues RM, Pereira AP, Fonseca SC, Gama SG, Theme MM Filha, et al. Trajetória das mulheres na definição pelo parto cesáreo: estudo de caso em duas unidades do sistema de saúde suplementar do estado do Rio de Janeiro. Cien Saude Colet. 2008;13(5):1521-34.

9. Robson MS. Classification of caesarean sections. Fetal Matern Med Rev. 2001;12(1):23-39.

10. Robson MS. Can we reduce the caesarean section rate? Best Pract Res Clin Obstet Gynaecol. 2001;15(1):179-94.

11. Abdel-Aleem H, Shaaban OM, Hassanin AI, Ibraheem AA. Analysis of cesarean delivery at Assiut University Hospital using the Ten Group Classification System. Int J Gynaecol Obstet. 2013;123(2):119-23.

12. Betrán AP, Vindevoghel N, Souza JP, Gülmezoglu AM, Torloni cesáreas $(25,2 \%)$, o que mostra que há a cultura de realizar cesáreas em pacientes com cesáreas anteriores;

- O grupo 2 teve a segunda maior contribuição (18,6\%) e neste grupo deve haver maior controle para reduzir a taxa de cesáreas da MEAC.

MR. A systematic review of the Robson Classification for cesarean section: what works, doesn't work and how to improve it. PLoS ONE. 2014;9(6):e97769.

13. Chong C, Su LL, Biswas A. Changing trends of cesarean section births by the Robson Ten Group Classification in a tertiary teaching hospital. Acta Obstet Gynecol Scand. 2012;91(12):1422-7.

14. Brennan DJ, Robson MS, Murphy M, O'Herlihy C. Comparative analysis of international cesarean delivery rates using 10-group classification identifies significant variation in spontaneous labor. Am J Obstet Gynecol. 2009;201(3):308.e1-8.

15. Ferreira EC. Utilização do sistema de classificação de dez grupos de Robson para partos na investigação de morbidade materna grave [dissertação]. Campinas: Universidade Estadual de Campinas; 2014. $88 \mathrm{p}$.

16. Ferreira EC, Pacagnella RC, Costa ML, Cecatti JG. The Robson ten group classification system for appraising deliveries at a tertiary referral hospital in Brazil. Int J Gynaecol Obstet. 2015;129(3):236-9.

17. Ferreira EC, Costa ML, Cecatti JG, Haddad SM, Parpinelli MA, Robson MS. Robson ten group classification system applied to women with severe maternal morbidity. Birth. 2015; 42(1):38-47.

18. Robson M, Murphy M, Byrne F. Quality assurance: the 10-group classification system (Robson classification), induction of labor, and cesarean delivery. Int J Gynaecol Obstet. 2015;131:S23-S27.

19. Brasil. Conselho Nacional de saúde. Resolução $n^{\circ} 466$, de 12 de dezembro de 2012. [Internet]. Brasília; 2012 [acesso em: 24 Jan 2016]. Disponível em: http://conselho.saude.gov.br/resolucoes/2012/ Reso466.pdf

20. World Health Organization. Appropriate technology for birth. Lancet. 1985; 2(8452):436-7.

21. Ye J, Betrán AP, Vela MG, Souza JP, Zhang J. Searching for the optimal rate of medically necessary cesarean delivery. Birth. 2014;41(3):237-44.

22. Kelly S, Sprague A, Fell DB, Murphy P, Aelicks N, Guo Y, et al. Examining caesarean Section rates in Canada using the Robson classification system. J Obstet Gynaecol Can. 2013;35(3):206-14.

23. Empresa Brasileira de Serviços Hospitalares. Relatório de gestão - produção assistencial 2015 [Internet]. Fortaleza: EBSERH; 2015 [acesso em: 09 fev 2016]. Disponível em: http://www.ebserh.gov.br/ documents/214336/0/COM+DEZEMBRO.pdf

\section{Como citar:}

Moura VA, Feitosa FE. Avaliação de cesáreas na Maternidade Escola Assis Chateaubriand utilizando o sistema de classificação de Robson em dez grupos. Rev Med UFC. 2017 jan-abr;57(1):25-29. 